\title{
Mass spectrometry imaging using dynamically harmonized FT-ICR at million resolving power: Rationalizing and optimizing sample preparation and instrumental parameters
}

Mathieu Tiquet ${ }^{1}$, Raphaël La Rocca', Daan van Kruining ${ }^{2}$, Pilar Martinez-Martinez ${ }^{2}$, Gauthier Eppe $^{1}$,Edwin De Pauw ${ }^{1}$, Loïc Quinton ${ }^{1}$, Johann Far ${ }^{1}$

${ }^{1}$ Mass Spectrometry Laboratory, MoISYS RU, University of Liège; Liège, Belgium

${ }^{2}$ Department of Psychiatry and Neuropsychology, School for Mental Health and Neuroscience, Maastricht University, Maastricht, the Netherlands

\section{Abstract}

MALDI mass spectrometry imaging (MSI) is a powerful analytical method giving access to the $2 D$ localizations of compounds in a thin section of a sample. To properly discern isobaric compounds in complex biological samples, dynamically harmonized ICR cell (ParaCell()) has been introduce to achieve extreme spectral resolution. However, in this work we show that high resolution MS images realized on a FTICR High resolution instrument with recommended parameters suffered from an abnormal shifting of $\mathrm{m} / \mathrm{z}$ ratios pixel to pixel. Resulting datasets show poor mass accuracy measurements and resolutions under manufacturer estimations. The abnormal mass shifting phenomenon has been linked to the stability of the Total Ion Current (TIC) during the image acquisition. This work proposes an optimization of laser parameters in order to limit the observed mass shift to retain machine specifications during MSI analyses. It is also shown that the method has been successfully employed to realize quality MS images with resolution above 1,000,000 in the lipid mass range across the whole image.

\section{Introduction}

Matrix-Assisted Laser Desorption/lonisation Mass Spectrometry Imaging (MALDI-MSI) has emerged as a label-free analytical method monitoring the relative abundance (neglecting the suppression of ionization) and spatial distribution for a wide variety of analytes, especially for biological samples $[1,2,3]$. To properly distinguish isobaric compounds [4] inherent to the complexity of biological samples, high resolving powers (R.P. > 300,000 @m/z: 400) and great mass measurement accuracies (MMA) are required. These performances are routinely attainable using an analyzer based on Fourier Transform-Ion Cyclotron Resonance (FT-ICR) [5]. Furthermore, recent developments introduced by E.N. Nikolaev and coworkers [6,7 \& 34] had seen emerged a dynamically harmonized ICR cell, commercialized by Bruker in the actual solariX XR brand mass spectrometer as ParaCell ${ }^{\circledR}$. This new cell provides even greater resolving power than ever before [8] 
(around 1,000,000 in the lipid mass range in broadband mode) and improved mass accuracies typically sub-ppm. These improvements are useful to increase the confidence level of the precursor ions empiric formula determination, especially when including the isotopic fine structure [9].

To obtain the highest quality level images by mass spectrometry (MS), each step of the mass spectrometry imaging workflow has to be properly optimized. Indeed from an instrumental point of view, significant deviations of the amount of injected ions between scan events affect the global performance of the dynamically harmonized ICR cell. The sample preparation of the sample also affects the ionization efficiency as well as the local diffusion of the analytes in tissue sections. Literature provides much information on various parameters like slice thickness[10-12], matrix and solvent choices [13-17]. More recently FT-ICR-MSI experimental optimization [18] is also available in the literature. However, when using recommended parameters and optimized methods intended for the previous ICR design "Infinity Cell $($ ", the best performance in R.P. and mass accuracy were far from machine specifications due to unusually strong mass shifts. This is mainly due to an excessively fluctuating ion current pixel from pixel during the MSI experiments. Usually, this phenomenon can be corrected using lock-mass calibration during acquisition $[19,20]$ which would ideally require several homogeneously distributed analytes. These targets could be added before matrix deposition at the risk of inducing more or less severe ion suppression effect(s). Another solution would be software post-acquisition recalibration [21] which could be time-consuming depending on the size and format of the dataset due to the added fomat conversion and computational steps.

This study reports and limits abnormal mass shifts observed during high/extreme resolution MALDI-FT-ICR-MSI fitted with the ParaCell®. The solution proposed here is solely based on sample preparation and acquisition method optimizations as a means to get images at an R.P. $>500.000$ @m/z: 800 (better than 1 million at m/z: 400) in broadband mode.

\section{Material and methods}

\subsection{Chemicals}

Acetone and methanol HPLC grade were obtained from Biosolve (Valkenswaard, Netherlands). Trifluoroacetic acid (TFA, 99\%) a-hydroxycinnamic acid (a-HCCA, purity 97\%), and red phosphorus $(\geq 97 \%)$ were purchased from Sigma-Aldrich (Taufkirchen, Germany). The internal standard SPLASH LipidoMIX ${ }^{\mathrm{TM}}$ containing deuterated lipids from several different families was purchased from Avanti Polar Lipids (Alabaster, Alabama, USA) via Sigma Aldrich. 


\subsection{Animal handling}

Transgenic mice were purchased from Dr. Mary Jo LaDu (University of Illinois at Chicago) and bred in-house at MHeNs at Maastricht University as described elsewhere [22]. In short, humanAPOE4 knock-in mice in which the mouse APOE gene was replaced by human APOE were crossbred with 5xFAD mice (Jackson laboratory) carrying human familial Alzheimer disease mutations PSEN1 and APP to obtain E4FAD mice with increased $A \beta$ peptide production $[22,23]$. Female E4FAD mice over 6 months of age were sacrificed by $\mathrm{CO} 2$ inhalation then brains were extracted. After extraction, mice brains were cut across the sagittal midline and immediately fresh-frozen in liquid nitrogen and subsequently stored at $-80{ }^{\circ} \mathrm{C}$. For transportation, samples were placed on dry ice and transferred to the University of Liège to be long-term stored conserved again at $-80^{\circ} \mathrm{C}$ before further handling. All procedures were approved by the Animal Welfare Committee of Maastricht University and were performed according to Dutch federal regulations for animal protection.

Natural AB-type zebrafish were bred in the GIGA at ULiège under the supervision of Pr. Marc Muller. The aquarium water was thermostated at $28^{\circ} \mathrm{C}$ with a circadian cycle of 14 hours of light and 10 hours of darkness. One-month-old fish were first anesthetized by adding tricaine mesylate to a concentration of $0.04 \%$ then increased to $0.16 \%$ in order to induce cardiac arrest. Fish were then embedded in gelatin $(350 \mathrm{mg} / \mathrm{mL})$ and stored at $-80^{\circ} \mathrm{C}$ for at least 24 hours. All procedures were approved by the Animal Welfare Committee of the University of Liège and were performed according to Belgian federal regulations for animal protection.

\subsection{Tissue sectioning}

Mouse brain tissues and zebrafish were sectioned into $14-\mu \mathrm{m}$ and $8-\mu \mathrm{m}$ thick sagittal slices respectively using a CryoStar NX70 (Thermo Fisher Scientific, Massachusetts, USA) set at $-20^{\circ} \mathrm{C}$. SEC35e low profile razor blades (Thermo Fisher Scientific, Massachusetts, USA) were employed at $15^{\circ} \mathrm{C}$ during the sectioning. Cryosections were thaw-mounted onto indium-tin-oxide coated conductive glass slides (Bruker Daltonics, Bremen, Germany).

\subsection{Matrix coating}

Before matrix deposition samples were dried in a vacuum desiccator for 15 minutes or until no visible wetness was observable. Dried samples were coated with matrix using the automatic sprayer SunCollect MALDI spotter (SunChrom, Friedrichsdorf, Germany). Matrix solution contained $5 \mathrm{mg} / \mathrm{mL}$ of $\alpha$-HCCA dissolved in methanol and milli-Q water acidified with TriFluoroacetic Acid 
(MeOH: $\mathrm{H}_{2} \mathrm{O}:$ TFA 9:0.9:0.1 v:v:v). During the spraying, the nozzle was positioned to its lowest setting and its moving speeds in the $X$ and $Y$ axis were set at medium $10(1540 \mathrm{~mm} / \mathrm{min})$. Used matrix flow rates started at $5 \mu \mathrm{L} / \mathrm{min}$ and were then increased to $10 \mu \mathrm{L} / \mathrm{min}$ after the third matrix deposition layer. The number of layers required to obtain a coating of roughly $10 \mathrm{nmol} / \mathrm{mm}^{2}$ of matrix was calculated for each matrix deposition with the spray. Later in this study, the amount of matrix deposited has been optimized to $5 \mathrm{nmol} / \mathrm{mm}^{2}$.

\subsection{MALDI mass spectrometry imaging}

Mass spectrometry acquisitions were performed on an ESI/MALDI dual source 9.4T MALDI-FTICR equipped with the ParaCell® (solariX XR 9.4T, Bruker Daltonics, Bremen, Germany) operating in MALDI positive mode with a data point size of 2,4 or $8 \mathrm{M}$ in the 300 to $1200 \mathrm{~m} / \mathrm{z}$ mass range. Other relevant parameters are listed in Table 1. Before acquisitions, the spectrometer was calibrated on the odd-numbered clusters of red phosphorus spotted close to the analyzed samples [24]. Automated acquisitions were performed using the software Flexlmaging 5.0 (Bruker Daltonics, Bremen, Germany) with a raster of $50 \mu \mathrm{m}$ in both axes.

Table 1 Sets of parameters used in the original and re-optimized method.

\begin{tabular}{|ll|l|l|}
\hline Parameters & $($ Unit $)$ & Original & $\begin{array}{c}\text { Re- } \\
\text { optimized }\end{array}$ \\
\hline Laser focus & & Minimum & Small \\
\hline Laser power & $(\%)$ & 60 & 60 \\
\hline Laser shots & & 600 & {$[2 ; 10]$} \\
\hline Laser frequency & $(\mathrm{Hz})$ & 1000 & \#shots ${ }^{\star} 10$ \\
\hline $\begin{array}{l}\text { Sweep excitation } \\
\text { power }\end{array}$ & $(\%)$ & 22 & {$[16 ; 18]$} \\
\hline $\begin{array}{l}\text { Front \& back trap } \\
\text { plate }\end{array}$ & $(\mathrm{V})$ & 1.5 & 1.35 \\
\hline Analyzer entrance & $(\mathrm{V})$ & -10 & {$[-5 ;-15]$} \\
\hline Side Kick & $(\mathrm{V})$ & 5 & {$[6 ; 10]$} \\
\hline Side Kick Offset & $(\mathrm{V})$ & -1.0 & -1.5 \\
\hline
\end{tabular}

\subsection{Data processing}

All datasets were visualized with SCiLS Lab 2016b (SCiLS, Bremen, Germany) after conversion into .sl format using the SQlite file generated by the instrument. Images shown were generated after total ion count normalization (unless specified otherwise) and automatic hot spot removal. Database bulk structure searches were done using the LIPID MAPS Structure Database (LMSD) tool offered by LIPID MAPS $\AA$ Lipidomics Gateway $[25,26]$. Queries were made on the full database with a $5 \mathrm{mDa}$ mass tolerance for $[\mathrm{M}+\mathrm{H}]^{+} ;\left[\mathrm{M}+\mathrm{H}-\mathrm{H}_{2} \mathrm{O}\right]^{+} ;[\mathrm{M}+\mathrm{Na}]^{+} ;[\mathrm{M}+\mathrm{K}]^{+}$; and $[\mathrm{M}+2 \mathrm{Na}-\mathrm{H}]^{+}$ions. Homemade script 
written in $\mathrm{R}$ language has been used to calculate the mass accuracies and resolving power standard deviations for given $\mathrm{m} / \mathrm{z}$ window in an MSI dataset converted in imzML format by FlexImaging 5.0.

\section{Results and discussion}

Ultra-High (or extreme) resolving power mass spectrometry images were acquired using an FTICR equipped with the dynamically harmonized ICR cell, also known as ParaCell@. The method employed consisted of recommended manufacturer MALDI MS values with minimal modifications to work in the 300 to 1200 amu mass range in broadband mode at an estimated resolving power (R.P.) above 400,000 at mass 800 . For each pixel, the resolution at full-width middle height (FWMH) was slightly above the estimated R.P by the FT-ICR control software (FTMS control). Nonetheless, the centroids of the $\mathrm{m} / \mathrm{z}$ peaks during the mass measurements were shifting beyond the specification of the solariX XR in term of mass accuracy. Consequently, the MSI mean spectrum showed widened peaks due to the spectrum-to-spectrum mass shifting which, in the most extreme cases, could even lead to peaks splitting by a few milli atomic mass units (i.e. several ppm above) as shown in Figure 1. The reconstructed mass spectrometry image of one of the detected ions results in biased and incomplete ion distributions unless the targeted ion and its shifted counterpart ion were selected (Erreur ! Source du renvoi introuvable.A and B). When comparing spectra from different pixels, it has been noticed that the Total lon Count (TIC) compared pixel-to-pixel in the mass spectrometry image was non-constant (Figure 1c) and that peaks splitting could be linked to the regions with lower or higher values of TIC (Figure 1d). We explain this result is due to large variations of TIC resulting in different space charge effects and/or Coulombic repulsion between the ion's packets inside the ICR cell. These fluctuations will influence the measured frequencies and thus mass over charge $(\mathrm{m} / \mathrm{z})$ ratios [27-29]. The work of Ferey et $a l^{18}$ already shows an acquisition method optimization for MALDIMSI using an FT-ICR equipped with a 12T magnet. However, even when using their optimized parameters, acquisition on our $9.4 \mathrm{~T}$ suffered from mass shift. It is worth noting that in theory, the usage of a higher-powered superconductive magnet would diminish the influence of the space charge effect as it is explained in Michael L.Easterling et al work [30-31]. A more powerful magnet would thus result in less mass shift but this aspect is not explored in this paper. In our case, the only way would be to reduce the fluctuation of the ion current during an acquisition to limit the observed mass shift. 
A. Image mean spetrum

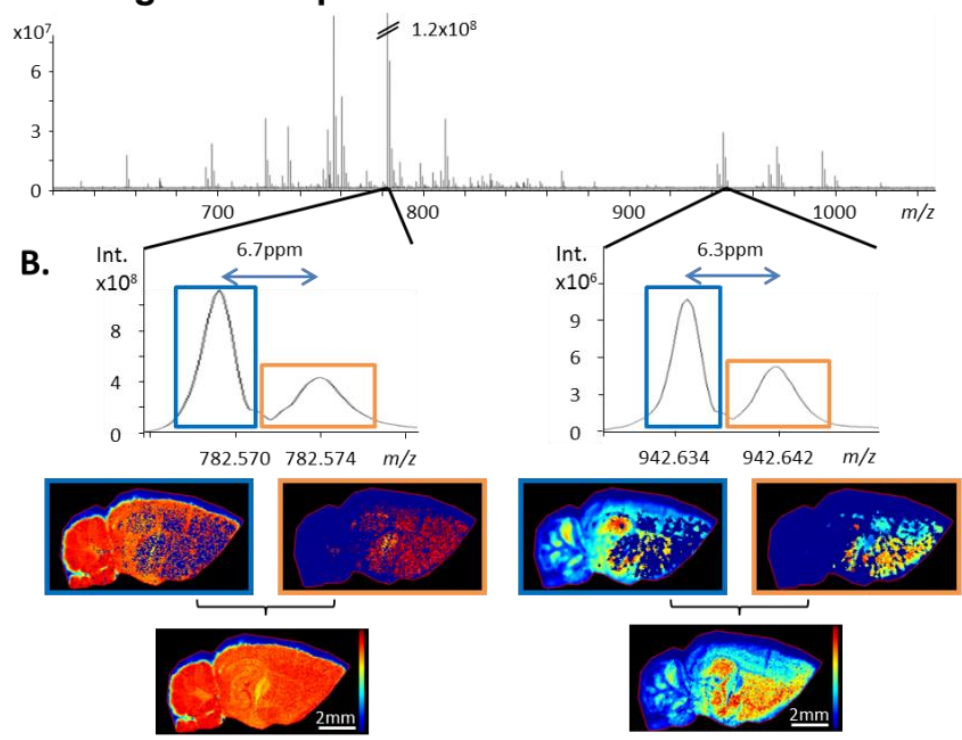

C. TIC heat map

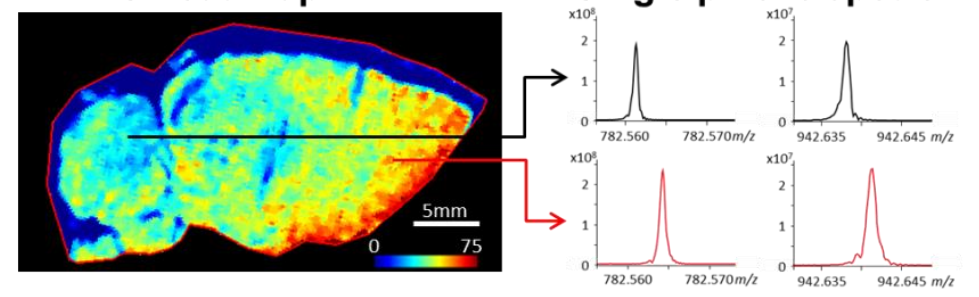

Figure 1 (a) Mean mass spectrum of the whole image of a rat brain slice analyzed by highresolution MALDI-FT-ICR-MSI SolariX XR 9.4T. (b) Zoomed mean mass spectrum focused on peaks $\mathrm{m} / \mathrm{z} 782.57$ and 942.64 showing artifacts of duplicated peaks and their incomplete distributions due to inconsistent mass measurement accuracy during the MSI acquisition. (c) Heat map of the non-normalized Total Ion Current of the MSI analysis (d) Mass spectra extracted from single pixels located in regions with significant differences in term Total lon Current.

In order to confirm the effect of large fluctuations of TIC values on the shifting of $m / z$ ratios, the TIC of deuterated lipids spotted on an ITO glass slide using HCCA as matrix was monitored. Before tests, the instrument was calibrated until [PC $(15: 0 / 18: 1(d 7))+H]^{+}$, [LysoPC $\left.(18: 1(d 7))+H\right]^{+}$and $[S M$ $(\mathrm{d} 18: 1 / 18: 1(\mathrm{~d} 9))+\mathrm{H}]^{+}$could be observed with resolutions $(\mathrm{FWMH})$ above 200,000 at their respective $\mathrm{m} / \mathrm{z}$ and MMA at $0.5 \mathrm{ppm}$ or better. Tests consisted of several scans over the sample showing small mass shifts and a rather stable TIC until the number of laser shots was manually increased to boost the number of ion in the ICR cells. This always resulted in a jump in mass for all ions ranging from 2 to 6ppm (Figure S 3) hinting to an implication of the TIC stability in the mass shift observed. From the MSI perspective, such variation of the TIC could appear due to a poor matric deposition creating hot 
spots or a naturally highly heterogeneous sample (i.e. tissue sections) showing region of interests with distinct TIC.

To better understand the influence of the TIC stability on the performance of the instrument during MSI acquisition, the relationship between the TIC value and the number of laser shots per scan has been investigated. Acquisitions of mass spectra summing up to 600 laser shots were made with the smallest possible increment at a fixed position on the same anatomical region of a mouse brain tissue section. Unfortunately, single laser shot acquisition showed no signal (not even the matrix). The method employed consisted of 2 laser shots per scan for 300 scans. The laser frequency also had to be decreased from $1000 \mathrm{~Hz}$ to $20 \mathrm{~Hz}$ due to an absence of signal above a certain threshold. Figure 2 shows corresponding results with a plot of TIC accumulation over time (Figure 2A) and TIC value for individual scans (Figure 2B). In Figure 2A it can be seen that most of the signal is obtained in the first 100 shots whereas Figure 2B shows a notably unstable TIC during the 50 firsts laser shots. When looking at mass spectra, the very few first shots show rich spectra in terms of peaks number. The next display only the most intense ions and finally only noise if the scans continue further. Figure $2 \mathrm{~A}$ also shows that first laser shots provide TIC raising to be rather linear followed by a slow down due to a lower amount of introduced ions newly generated by MALDI. Then a new linear increase is observed after roughly 120 laser shots which is only due to the peak noise. This means that unless a particular target ion requires a large amount of laser energy to be detected, a lower amount of laser shots would be beneficial when more stable TIC is required. Thus for further extreme resolution imaging, fewer laser shots will be performed to stay in the early linear section of the curve in Figure $2 \mathrm{~A}$ without compromising the molecular register of the mass spectrometry image. By staying in this zone we should increase the repeatability of the ionization process to ensure a stable amount of ions injected into the ICR cell and limit the TIC fluctuation during extreme resolution MSI.

A.

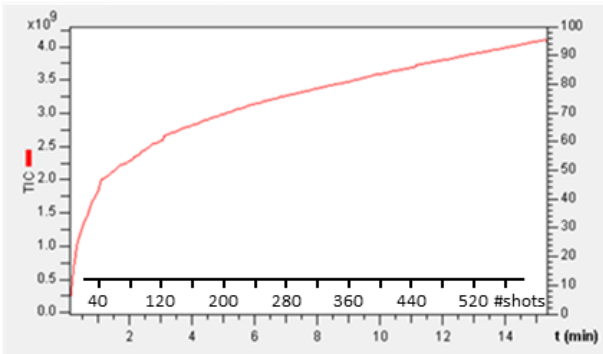

B.

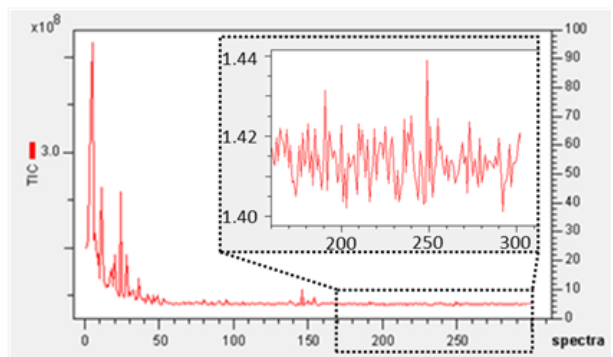

Figure 2 Graphs of the TIC accumulation over time (a) and the Total lon Current per scan (b) for a 300 scans acquisition of 2 laser shots at $20 \mathrm{~Hz}$ per scan realized on a dynamically harmonized MALDI-FT-ICR solariX XR 9.4T. 
Ion optic voltages of the ICR mass analyzer were also investigated as a potential factor for TIC stabilization. Screened voltages were analyzer entrance, front and back trapping, sidekick, and excitation sweep. Out of those parameters, only the sidekick was found to have a slight influence on the mass shift while not having a drastic effect on the TIC. Yet, the optimization of this parameter alone was not enough to reduce the observed mass shift to machine specifications. The voltage of the analyzer entrance had an interesting effect worth noticing. While slightly tweaking this parameter didn't seem to have any effect. Interestingly if the analyzer entrance voltage is reduced enough, the TIC would slowly start to reduce until no signal could be obtained. This could be useful to limit on realtime the quantity of ion entering the ICR cell by monitoring the mirror current generated in source, and help the analysis of highly heterogeneous samples and tissue sections. Of course, it would require further study and is currently seen as a double-edged sword. Investigation of front \& back trapping and excitation sweep lead to better peak shapes when properly optimized.

To evaluate the performance of an acquisition method with fewer laser shots, seriated sagittal mouse brain slices were prepared and paired slices were coated with the same matrix spray. The amount of deposited MALDI matrix was investigated in terms of signal suppression of the analytes of interest which resulted in spraying half of the usual $10 \mathrm{nmol} / \mathrm{mm}^{2}$ sprayed. Images were acquired with 600 laser shots at $1000 \mathrm{~Hz}$ (Figure 3A "Old") for one and 6 laser shots at $60 \mathrm{~Hz}$ for the other (Figure $3 \mathrm{~A}$ "New"). Note that for the second image (i.e. Figure 3A "New"), the laser focus was also modified from minimum to small to compensate for the smaller zone irradiated. Figure $3 \mathrm{~A}$ "Old" show unstable TIC for the method employing the non-optimized parameters while the new set of optimized settings showed a drastically improved TIC stability through the entire acquisition (Figure 3B "New") which resulted in mass shifts restrained below $0.5 \mathrm{ppm}$ and even increased resolution in the mean spectrum (Figure 3C) for the detected ions. To quantify the effect of TIC stability, homemade software has been used to follow $\mathrm{m} / \mathrm{z}$ channels for every pixel to calculate the distribution of their mass (apex of the $\mathrm{m} / \mathrm{z}$ peaks) and the resolving power in terms of full width at half maximum. Graphical representation of the mass and resolving power distribution for the ion $782.57 \mathrm{~m} / \mathrm{z}$ are provided in supporting information (Figure S1\&2). 

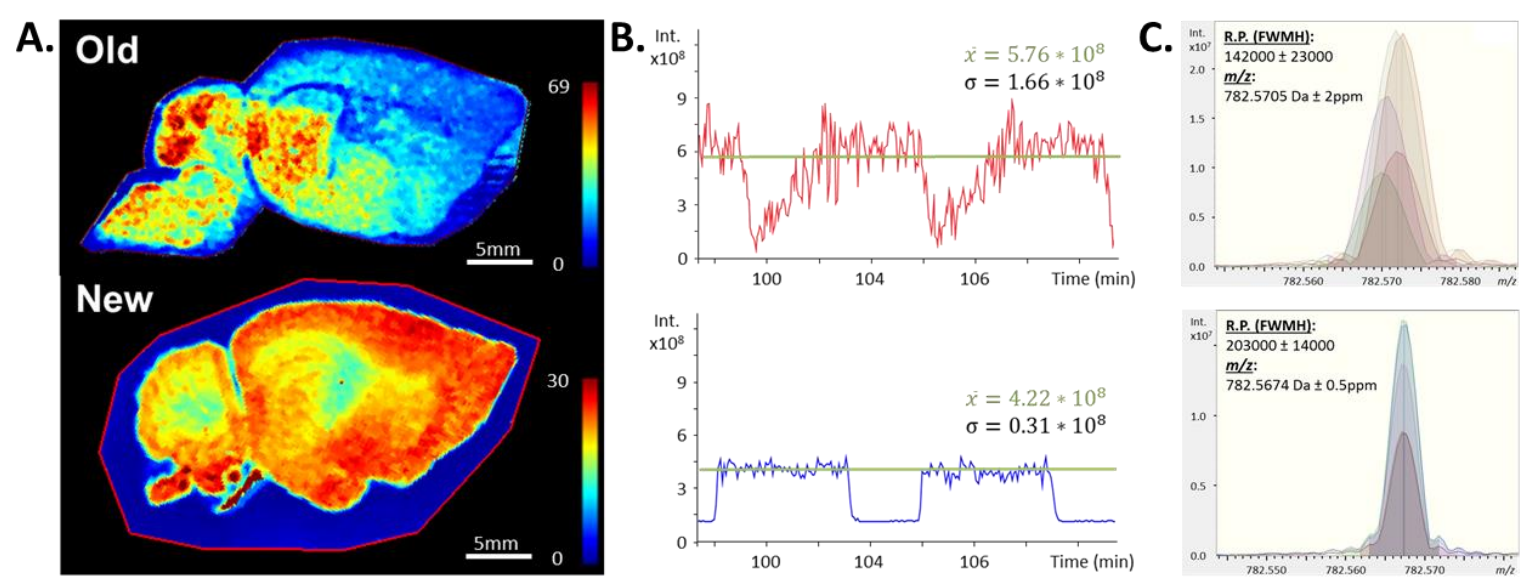

Figure 3 (a) Heat map of the non-normalized Total Ion Count of dynamically harmonized MALDI-FT-ICR-MSI solariX XR 9.4T acquired with manufacturer recommendation based method (up) and lower amount of laser shots based method (down) See text for details. (b) Portions of the TIC over time of MSI acquisition and the calculated mean intensities and standard deviation (values from pixel outside of the sample were excluded) (c) Multi-pixel spectrum overlay of $\mathrm{m} / \mathrm{z} 782.57$ shows the notable improvement in terms of resolving power and mass accuracy measurements during the mass spectrometry images between the unstable (up) and stable (down) Total Ion Count.

Drastically reducing the amount of laser shot limited the overall signal in the mass spectra (TIC) as expected while the detected ions in the mass spectra for both methods were comparable. The $\mathrm{m} / \mathrm{z}$ not detected any more being peaks already close to the limit of detection and isotope contribution. Interestingly, when using fewer laser shots, the absolute intensities of minor ions were reduced in a smaller proportion than were major ions. FT-ICR manual acquisitions were performed on a brain sample to compare a 10 laser shots versus 100 shots at $100 \mathrm{~Hz}$ on the same anatomical region of the brain tissue to support our statement. Table 2 reports data of some lipids identified by database matches that highlight this observation. Ion 772.53 and 798.54 are the most intense in the spectra while targets $\mathrm{m} / \mathrm{z} 770.51$ and 848.56 are selected among the less intense ions of the spectrum. When comparing 10 to 100 laser shots, intensities of minor ions are seen to be roughly halved while major ions intensities decrease by an order of magnitude. While using 100 laser shots the intensity ratio of 798.54 over 770.51 reached 40 but decrease to a value of only 11.3 when using 10 laser shots. This shows that most intense ions had their intensity decreasing in a larger proportion compared to less intense ion as the same observation can be made with any combination of an intense ion over a less intense one. Furthermore, we see that the intensity ratio in not that impacted by the amount of laser shot per scan between ions of comparable intensities ( 772.53 vs 798.54$)$. These results were in total 
agreement with a MALDI-ToF instrument (rapifleX, Bruker, Germany) used for comparison but only when the beamscan option was not activated as it would distribute the laser shots over the selected surface area ensuring fresh matrix for method with higher number of laser shots (i.e using matrix blaster). In brief, the representativity of the minor ions when using lower laser shots was not instrument dependent but reflected the MALDI ionization mechanisms of these ions in the tissue sections, which will be very interesting to investigate further.

Table 2 Identification of several ion and their intensities depending on the number of laser shots realized acquired on a mouse brain sample with a MALDI-FT-ICR instrument.

\begin{tabular}{|c|c|c|c|c|c|c|c|c|c|}
\hline Target $\mathrm{m} / \mathrm{z}$ & Identification & $\begin{array}{l}10 \text { shots } \\
\text { Intensity }\end{array}$ & $\begin{array}{c}10 \text { shots } \\
\text { Mass accuracy } \\
(\mathrm{ppm})\end{array}$ & $\begin{array}{l}100 \text { shots } \\
\text { Intensity }\end{array}$ & $\begin{array}{c}100 \text { shots } \\
\text { Mass accuracy } \\
(\mathrm{ppm})\end{array}$ & $\begin{array}{c}10 \text { shots } \\
\text { Ratio of } \mathrm{m} / \mathrm{z} \\
772.53 \text { over } \\
\text { target } \mathrm{m} / \mathrm{z}\end{array}$ & $\begin{array}{c}100 \text { shots } \\
\text { Ratio of } \mathrm{m} / \mathrm{z} \\
772.53 \text { over } \\
\text { target } \mathrm{m} / \mathrm{z}\end{array}$ & $\begin{array}{c}10 \text { shots } \\
\text { Ratio of } \mathrm{m} / \mathrm{z} \\
798.54 \text { over } \\
\text { target } \mathrm{m} / \mathrm{z}\end{array}$ & $\begin{array}{c}100 \text { shots } \\
\text { Ratio of } \mathrm{m} / \mathrm{z} \\
798.54 \text { over } \\
\text { target } \mathrm{m} / \mathrm{z}\end{array}$ \\
\hline 770.50975 & [PA 36:2+K]+ & $4.80 \mathrm{E}+05$ & -0.09 & $1.30 \mathrm{E}+06$ & -0.12 & 10.6 & 0.03 & 11.3 & 40.0 \\
\hline 848.55643 & {$[\mathrm{PC} 38: 4+\mathrm{K}]+$} & $8.90 \mathrm{E}+05$ & -0.25 & $6.50 \mathrm{E}+06$ & -0.24 & 5.7 & 0.15 & 6.1 & 8.0 \\
\hline 772.52519 & {$[P C 32: 0+K]+$} & $5.10 \mathrm{E}+06$ & -0.13 & $4.30 \mathrm{E}+07$ & -0.17 & - & - & 1.06 & 1.21 \\
\hline 798.54079 & {$[P C 34: 1+K]+$} & $5.40 \mathrm{E}+06$ & -0.26 & $5.20 \mathrm{E}+07$ & -0.26 & 0.94 & 0.83 & - & - \\
\hline
\end{tabular}

When applying the revisited method for extreme resolution MSI with an estimated R.P. over $500,000 @ m / z 800$, a mass shift with a much lower amplitude than before is observed and now fitting with the instrument specification. The FWMH resolutions in individual spectra are now similar to the one in the mean spectrum. When comparing the mean spectrum of centroided MSI data performed on seriated brain slices (Figure 4a) and whole-body zebrafish slices (Figure 4b) the difference in image quality becomes obvious. The new method with controlled ion injection in the cell (TIC control) provided thinner $\mathrm{m} / \mathrm{z}$ peaks due to restrained mass shift amplitude. The ion distribution in the tissue section of ions is also cleaner whatever TIC or RMS normalization is used or not (RMS not showed). To demonstrate the robustness of the controlled TIC method, a triplicate of brain seriated slices (roughly 12,000 pixels) and zebrafish whole body seriated slices (roughly 20,000) were acquired using the new method. Triplicates showed resolutions matching with the resolving power expected by the acquisition software, showing promise for the repeatability of the method. The imaging method has been tested for images at an FWMH resolution above 1,000,000@m/z 800 on the brain of a zebrafish slice (roughly 1500 pixels) which is available in the supporting information (Figure S3). This image obtained was of good quality and allows to observe the isotopic fine structure of intense ions in the mean spectrum (see SI). However, these images still had a slight loss of mass resolving power in the mean spectrum compared to individual pixels which is understandable as a measured difference 
of 0.4 milli atomic mass unit (corresponding machine specification of $0.5 \mathrm{ppm}$ at $\mathrm{m} / \mathrm{z} 800$ ) between two pixels is already enough to produce a slight peak split in the mean spectrum. Nonetheless, the isotopic fine structure in one pixel of interest is achieved. Note that a peak realignment strategy by software post-processing coupled with our proposed MSI method of the mass spectrometry images is developed in our group and restore the isotopic fine structure in the mean spectrum [32, 33].

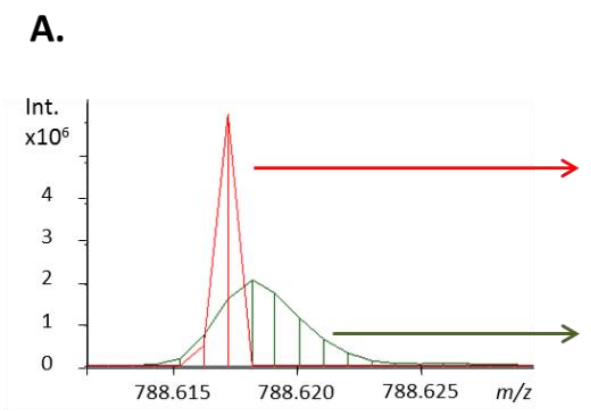

B.
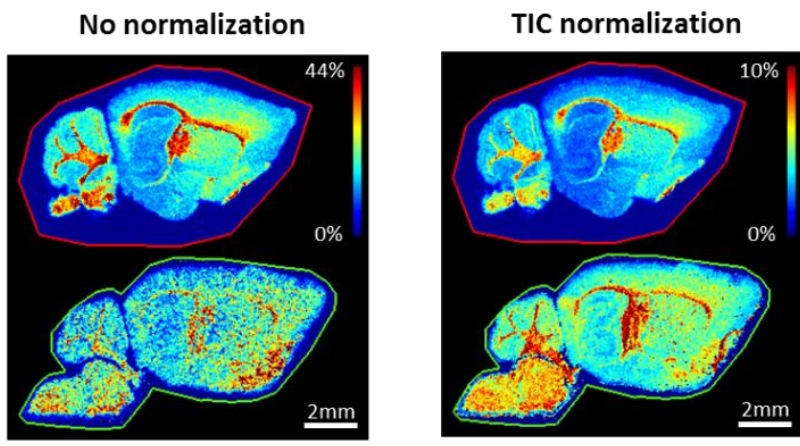

TIC control No TIC control
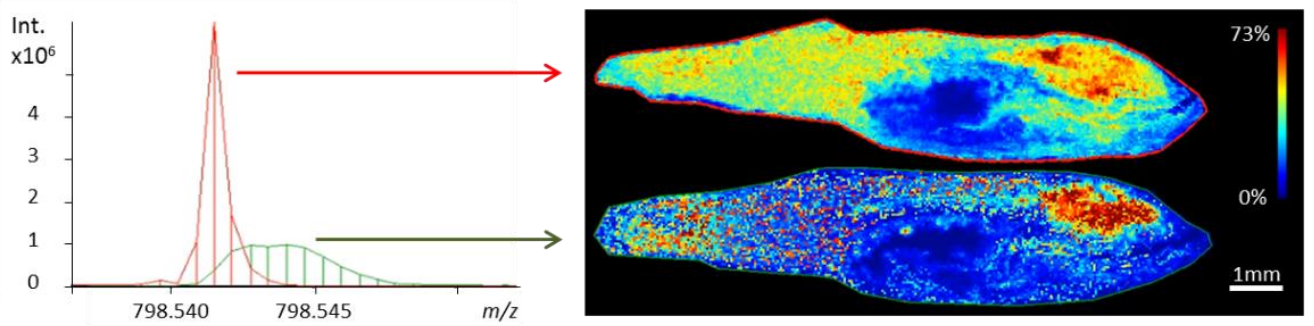

TIC control

No TIC control

Figure 4 (a) Averaged mass spectra of MALDI-FT-ICR (solariX XR 9.4T, fitted with ParaCell) of mouse brain (a) and zebrafish (b) zoomed on peak $\mathrm{m} / \mathrm{z} 788,62$ and 798.54 respectively, showing the width differences due to mass measurement accuracy obtained with (red) and without (green) TIC control and the distribution of $\mathrm{m} / \mathrm{z} 788.618 \pm 0.003$. Our proposed method produced sharper mass spectrometry images with significantly improved resolving power.

Lastly, database queries for mass lists obtained with our new method showed better peak annotation and resulted in less false positives, fewer false negatives, and better database matches due to the improved mass resolving power and mass accuracy. Figure 5 shows examples of database results as histograms of the matching counts at a given mass accuracy in ppm to make the detection of any oddities in the dataset easier. In the case were the TIC was not stable, most of the identifications had an error of around $-1.5 \mathrm{ppm}$ which is not consistent with the specification of an FTICR properly calibrated and poorly match the required mass accuracy for proper annotation of lipids from the Lipidmaps database. In comparison, the image with the stable TIC has most of its database matches scoring with $+0.4 \mathrm{ppm}$ which was expected due to the nominal performance for the 
instrument in terms of mass accuracy. Also, the number of total matches is improved due to fewer false-negative results and similarly potentially fewer false-positive results.

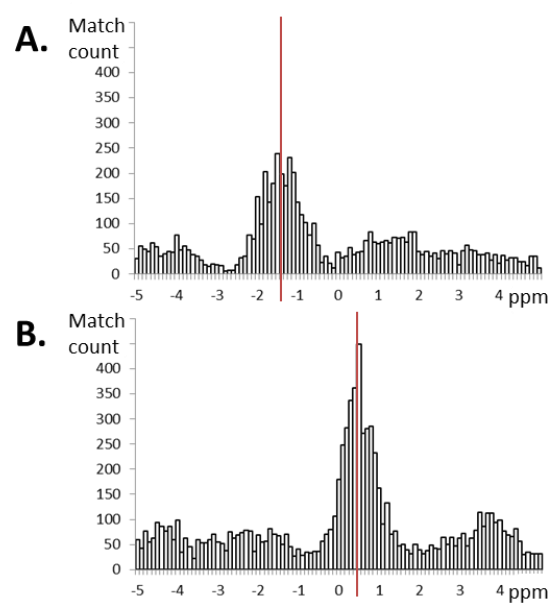

Figure 5 Representations of LIPID MAPS structure database (LMSD) bulk search results for peak lists as the number of matches versus their respective error in ppm. MALDI dynamically harmonized FT-ICR MSI datasets shown were acquired with unstable Total Ion Count (A) and stabilized Total Ion Current (B).

\section{Conclusion}

We successfully improved the mass shift and the mass accuracy of MALDI mass spectrometry images of mouse brain and Zebrafish tissue sections by introducing a controlled TIC injection method in the ICR cell of our solariX XR 9.4T, a dual-source ESI/MALDI fitted with the recently introduced Paracell in a commercially available instrument. Mass spectrometry image having a resolving mass power better than $11^{\prime} 000$ '000 at $\mathrm{m} / \mathrm{z} 800$ were successfully produced with no more mass shift than $+0.5 \mathrm{ppm}$ (0.4 milli atomic mass unit). The resulting images were also sharper at constant laser lateral resolution and matrix deposition method. In conclusion, we show that MS-images with extremes resolutions using limited power of magnetic fields produced by supraconductor magnets (below 12T) require a better control over the TIC through the acquisition to keep a good quality of information. Peak annotation using Lipidmaps database were matching with scores better $0.4 \mathrm{ppm}$, limiting the misidentification of the detected lipid in the images. This has been reached by revisiting mostly the laser parameters to improve method reproducibility pixels to pixels and limiting the mass shift of signal that prevented the loss of mass resolving power in an image mean spectrum. The robustness of our method was tested by performing mass spectrometry images of tissue section in triplicates with success. Despite it is out-of-scope of this paper, our data also provided interesting data in terms of 
MALDI ionization mechanisms when looking at the minor versus major lipid signals in tissue sections which require further investigation.

\section{Acknowledgments}

This work was supported by the Interreg euregio Meuse-Rhin project EurLipids. Many thanks to Pr. Martinez and her team for providing the mouse brain samples required for this study. All experiments were done with permission from the Committee on Animal Welfare of Maastricht University or from the Committee on Animal Welfare of Liege University, according to Dutch or Belgian governmental rules respectively. This work was also a contribution for the EU Horizon 2020 research and Innovation program under grant agreement № 731077 and the European Project EU_FT-ICR_MS (H2020 INFRAIA-02-2017). The MALDI TOF rapifleX and the MALDI FT-ICR solariX XR were co-funded by FEDER BIOMED HUB Technology Support (number 2.2.1/996).

\section{References}

(1) Le Rhun, E.; Duhamel, M.; Wisztorski, M.; Gimeno, J. P.; Zairi, F.; Escande, F.; Reyns, N.; Kobeissy, F.; Maurage, C. A.; Salzet, M.; Fournier, I. Biochim. Biophys. Acta - Proteins Proteomics 2017, 1865, 875-890.

(2) Ellis, S. R.; Cappell, J.; Potočnik, N. O.; Balluff, B.; Hamaide, J.; Van Der Linden, A.; Heeren, R. M. A. Analyst 2016, 141, 3832-3841.

(3) Lamont, L.; Eijkel, G. B.; Jones, E. A.; Flinders, B.; Ellis, S. R.; Porta Siegel, T.; Heeren, R. M. A.; Vreeken, R. J. Anal. Chem. 2018, 90, 13229-13235.

(4) Wang, J.; Wang, C.; Han, X. Anal. Chim. Acta 2019, 1061, 28-41.

(5) Marshall, A. G.; Hendrickson, C. L. Annu. Rev. Anal. Chem. 2008, 1, 579-599.

(6) Kostyukevich, Y. I.; Vladimirov, G. N.; Nikolaev, E. N. J. Am. Soc. Mass Spectrom. 2012, 23, 2198-2207.

(7) Nikolaev, E. N.; Kostyukevich, Y. I.; Vladimirov, G. N. Mass Spectrom. Rev. 2014.

(8) Jertz, R.; Friedrich, J.; Kriete, C.; Nikolaev, E. N.; Baykut, G. J. Am. Soc. Mass Spectrom. 2015, 26, 1349-1366.

(9) Popov, I. A.; Nagornov, K.; N.vladimirov, G.; Kostyukevich, Y. I.; Nikolaev, E. N. J. Am. Soc. Mass Spectrom. 2014, 25, 790-799.

(10) Longuespée, R.; Kriegsmann, K.; Cremer, M.; Zgorzelski, C.; Casadonte, R.; Kazdal, D.; Kriegsmann, J.; Weichert, W.; Schwamborn, K.; Fresnais, M.; Schirmacher, P.; Kriegsmann, M. Proteomics - Clin. Appl. 2019, 13, 1-7.

(11) Goodwin, R. J. A. J. Proteomics 2012, 75, 4893-4911.

(12) Shimma, S.; Sugiura, Y. Mass Spectrom. 2014, 3, S0029-S0029.

(13) Morikawa-Ichinose, T.; Fujimura, Y.; Murayama, F.; Yamazaki, Y.; Yamamoto, T.; Wariishi, H.; Miura, D. J. Am. Soc. Mass Spectrom. 2019.

(14) Kaletaş, B. K.; Van Der Wiel, I. M.; Stauber, J.; Dekker, L. J.; Güzel, C.; Kros, J. M.; Luider, T. M.; Heeren, R. M. A. Proteomics 2009, 9, 2622-2633.

(15) Grassl, J.; Taylor, N. L.; Millar, A. H. Plant Methods 2011, 7, 1-11. 
(16) Nishidate, M.; Hayashi, M.; Aikawa, H.; Tanaka, K.; Nakada, N.; Miura, S. ichi; Ryu, S.; Higashi, T.; Ikarashi, Y.; Fujiwara, Y.; Hamada, A. Drug Metab. Pharmacokinet. 2019, 34, 209-216.

(17) Prideaux, B.; Stoeckli, M. J. Proteomics 2012, 75, 4999-5013.

(18) Ferey, J.; Marguet, F.; Laquerrière, A.; Marret, S.; Schmitz-Afonso, I.; Bekri, S.; Afonso, C.; Tebani, A. Anal. Bioanal. Chem. 2019, 411, 3891-3903.

(19) Römpp, A.; Guenther, S.; Schober, Y.; Schulz, O.; Takats, Z.; Kummer, W.; Spengler, B. Angew. Chemie - Int. Ed. 2010, 49, 3834-3838.

(20) Barry, J. A.; Robichaud, G.; Muddiman, D. C. J. Am. Soc. Mass Spectrom. 2013, 24, 11371145.

(21) Smith, D. F.; Kharchenko, A.; Konijnenburg, M.; Klinkert, I.; Paša-Tolić, L.; Heeren, R. M. A. J. Am. Soc. Mass Spectrom. 2012, 23, 1865-1872.

(22) Youmans, K. L.; Tai, L. M.; Nwabuisi-Heath, E.; Jungbauer, L.; Kanekiyo, T.; Gan, M.; Kim, J.; Eimer, W. A.; Estus, S.; Rebeck, G. W.; Weeber, E. J.; Bu, G.; Yu, C.; LaDu, M. J. J. Biol. Chem. 2012, 287, 41774-41786.

(23) Oakley, H.; Cole, S. L.; Logan, S.; Maus, E.; Shao, P.; Craft, J.; Guillozet-Bongaarts, A.; Ohno, M.; Disterhoft, J.; Van Eldik, L.; Berry, R.; Vassar, R. J. Neurosci. 2006, 26, 10129-10140.

(24) Sládková, K.; Houška, J.; Havel, J. Rapid Commun. Mass Spectrom. 2009, 23, 3114-3118.

(25) Fahy, E.; Sud, M.; Cotter, D.; Subramaniam, S. Nucleic Acids Res. 2007, 35, 606-612.

(26) Sud, M.; Fahy, E.; Cotter, D.; Brown, A.; Dennis, E. A.; Glass, C. K.; Merrill, A. H.; Murphy, R. C.; Raetz, C. R. H.; Russell, D. W.; Subramaniam, S. Nucleic Acids Res. 2007, 35, 527-532.

(27) Gorshkov, M. V; Marshall, A. G.; Nikolaev, E. N. 1993.

(28) Masselon, C.; Tolmachev, A. V; Anderson, G. A.; Harkewicz, R.; Smith, R. D. 2002, 0305.

(29) Busch, K. Spectroscopy 2004, 19, 35-38.

(30) Francl, T. J.; Sherman, M. G.; Hunter, R. L.; Locke, M. J.; Bowers, W. D.; Mclver, R. T. Int. J. Mass Spectrom. Ion Processes 1983, 54, 189-199.

(31) Michael L. Easterling, Todd H. Mize, and I. Jonathan Amster. Anal. Chem. 1999, 71, 624-632

(32) La Rocca, Raphaël; Kune, Christopher; Tiquet, Mathieu; Stuart, Lachlan; Alexandrov, Theodore; De Pauw, Edwin; et al. (2020): Using Biological Signals for Mass Recalibration of Mass Spectrometry Imaging Data. ChemRxiv. Preprint. https://doi.org/10.26434/chemrxiv.12901679.v1

(33) $A B C$ paper to add

(34) Boldin, I.A.; Nikolaev, E.N. Rapid Commun. Mass Spectrom. 2011, 25, 122-126 


\section{Supporting information}

\section{Mass spectrometry imaging using dynamically harmonized FT-ICR at million resolving power: Rationalizing and optimizing sample preparation and instrumental parameters}

Mathieu Tiquet ${ }^{1}$, Raphaël La Rocca', Daan van Kruining ${ }^{2}$, Pilar Martinez-Martinez ${ }^{2}$, Gauthier Eppe $^{1}$, Edwin De Pauw ${ }^{1}$, Loïc Quinton ${ }^{1}$, Johann Far $^{1}$

${ }^{1}$ Mass Spectrometry Laboratory, MoISYS RU, University of Liège; Liège, Belgium

${ }^{2}$ Department of Psychiatry and Neuropsychology, School for Mental Health and Neuroscience, Maastricht University, Maastricht, the Netherlands

Figure $S 1$ Distribution of mass to charge ratio values found in the window $782.570+10 \mathrm{mTh}$ for the original acquisition method (left) and the method modified to stabilize TIC through MSI acquisition (right).

Figure S 2 Distribution of the resolving power of signal found in the window $782.570+10 \mathrm{mTh}$ for the original acquisition method (left) and the method modified to stabilize TIC through MSI acquisition (right).

Figure S 3 TIC vs time of a MALDI-FT-ICR acquisition where an increase of ions injected in the dynamically harmonized ICR cell is artificially induced around 3 minutes (A). Observable mass shift between the mass spectra recorded before (blue) and after (red) the TIC increase shown in part (B).

Figure S 4 Average mass spectrum of an MSI experiment with more than 2000 pixels at ultra high resolution in the 400 to 1200 mass range (up). Zoom on the different isotopes of the most intense ion where the isotopic fine structure is observed (middle). Distribution of ion 798.5417 in the brain of a zebrafish (bottom left). Result from a database search in LipidMaps showing all matches with a 0.005 Th tolerance (bottom right). 

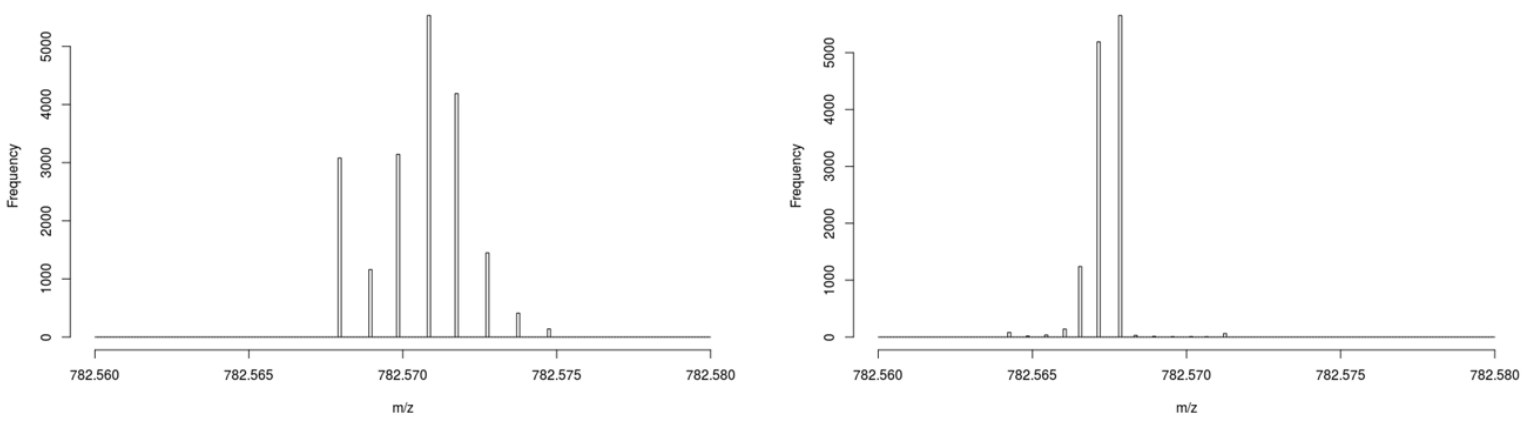

Figure $\mathbf{S} 1$ Distribution of mass to charge ratio values found in the window $782.570+10 \mathrm{mTh}$ for the original acquisition method (left) and the method modified to stabilize TIC through MSI acquisition (right).
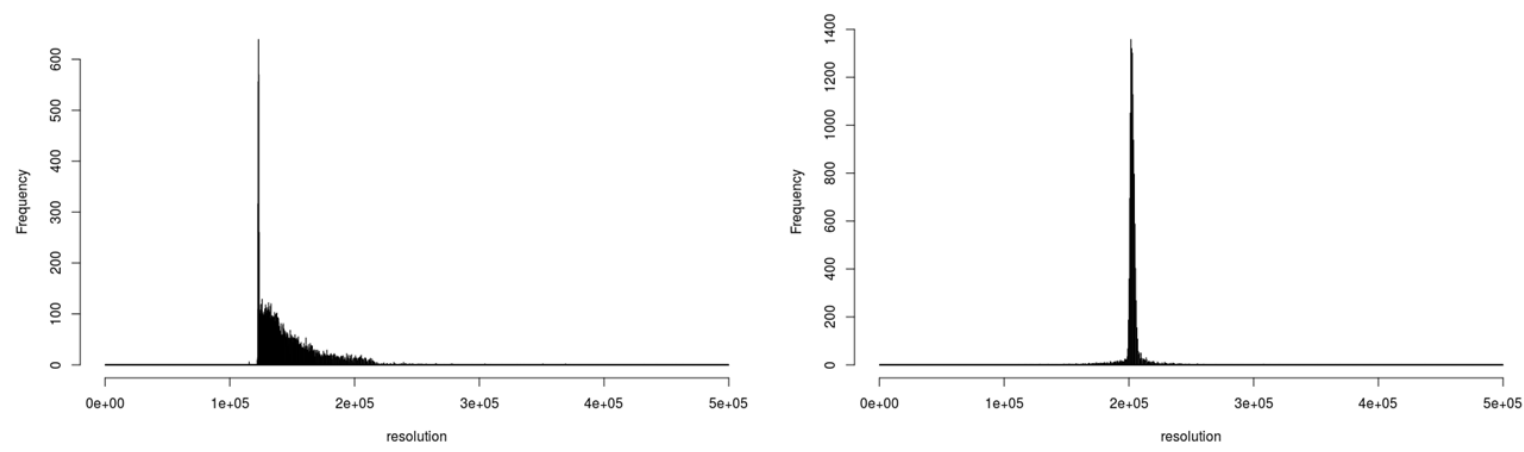

Figure S 2 Distribution of the resolving power of signal found in the window $782.570+10 \mathrm{mTh}$ for the original acquisition method (left) and the method modified to stabilize TIC through MSI acquisition (right). 

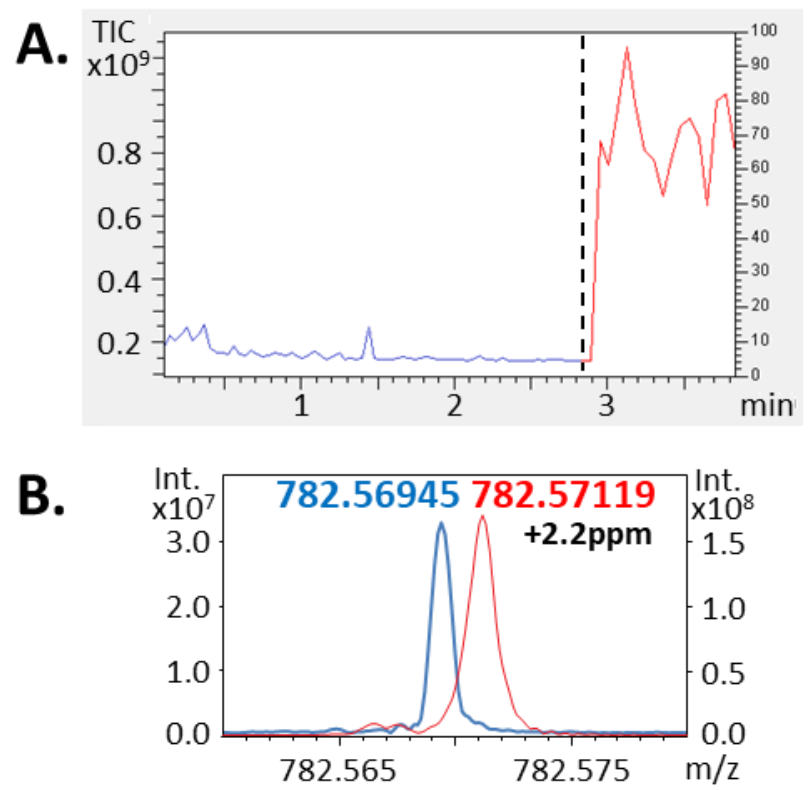

Figure S 3 TIC vs time of a MALDI-FT-ICR acquisition where an increase of ions injected in the dynamically harmonized ICR cell is artificially induced around 3 minutes (A). Observable mass shift between the mass spectra recorded before (blue) and after (red) the TIC increase shown in part (B). 

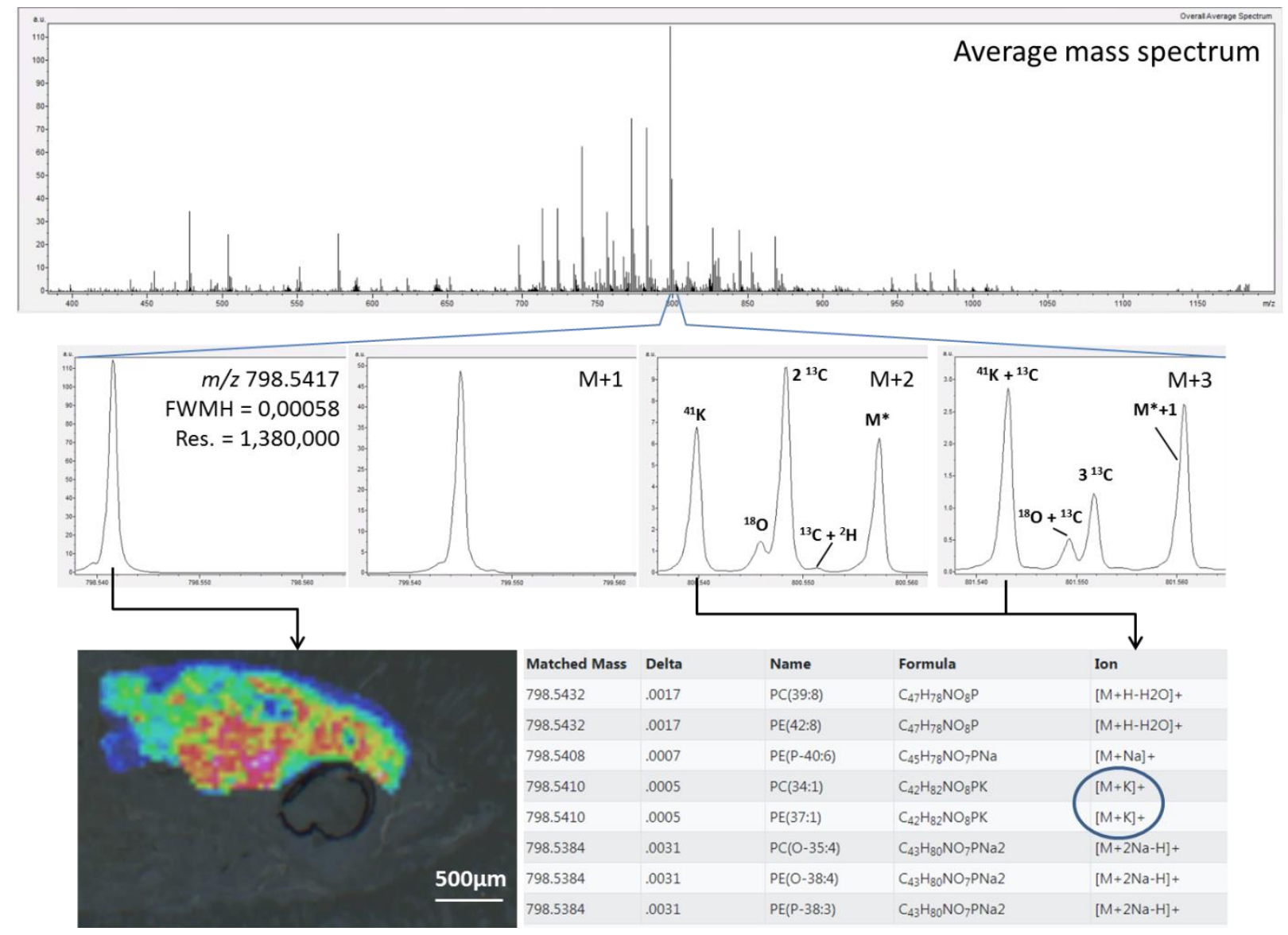

Figure S 4 Average mass spectrum of an MSI experiment with more than 2000 pixels at ultra high resolution in the 400 to 1200 mass range (up). Zoom on the different isotopes of the most intense ion where the isotopic fine structure is observed (middle). Distribution of ion 798.5417 in the brain of a zebrafish (bottom left). Result from a database search in LipidMaps showing all matches with a 0.005 Th tolerance (bottom right). 